\title{
Milk Purity Recognition Software through Image Processing
}

\author{
Alvarado-Díaz Witman ${ }^{1}$, Meneses-Claudio Brian², Roman-Gonzalez Avid ${ }^{3}$ \\ Image Processing Research Laboratory (INTI-Lab) \\ Universidad de Ciencias y Humanidades (UCH), Lima, Peru
}

\begin{abstract}
Currently in Peru, there is a per capita milk consumption of $87 \mathrm{~kg}$ per year; however, the Food and Agriculture Organization of the United Nations (FAO) recommends a consumption of $120 \mathrm{~kg}$ per person; the industry, when the milk is acquired from small livestock suppliers, does not analyze the milk before buying it, which there is a high risk that the milk is adulterated with water, in this sense, it proposes an alternative way of preliminary detection of the presence of water in milk, only through a laser a photograph, which greatly reduces the costs of milk analysis. Milk contains different nutrients, vitamins and minerals, which are beneficial for people, so it is very known if it is adulterated or not, that way to prevent diseases. In this document, the reader will read an alternative to the existing methods for the analysis of milk, for the presented method the application of Matlab Classification Learner and the fine K-Nearest Neighbors (KNN) algorithm were used, in which a success rate of $95.4 \%$ was obtained.
\end{abstract}

Keywords-Milk; adulterated milk; milk with water; milk analysis; image processing; classification learner; image processing

\section{INTRODUCTION}

One of the objectives of the Ministerio de Agricultura y Riego (MINAGRI) for the year 2021, is to achieve a national production of raw milk of 2.7 million tons, with an average yield of $9.8 \mathrm{~kg}$ per day per cow and a per capita consumption of $120 \mathrm{~kg}$ per person per year. Milk is an essential food, since it contains proteins of high biological value, between 3 and $5 \%$, it also provides an important percentage of fundamental elements such as potassium, phosphorus, Vitamins A, B, B2, $\mathrm{D}$, Niacin, necessary fats and minerals such as magnesium, potassium, zinc and phosphorus.

There are different works to verify if there are adulterated material in the milk, in [1], [2], [3] and [4] it is carried out through the study of the impedance, using different techniques such as the use of MSNC (Minimum Square Nonlinear Complex), KNN (K-Nearest Neighbors), LDA (Linear Discriminant Analysis), respectively, with papers of this type, adulterated milk has been identified with an approximate efficiency of $94.9 \%$.

In the research [5] they mention that in countries like India, adulteration in milk implies health problems, and even corruption, they also mention that there are compounds that can adulterate milk such as: water, flour, starch and even urea in undetectable measures. In the work, a low-cost optofluidic microviscometer is described, whose operation is based on the linear relationship between the dynamic viscosity and the width of the channel derived from the flow of two immiscible fluids within a channel, based on the operating principle of the Hagen-Poiseuille flow equation. In the study, about 60 milk samples with various adulteration ratios were analyzed. It was finally found that the device was accurate enough to measure the entire range of adulteration ratios with $95 \%$ accuracy.

In [6], they present a hardware platform based on Near Infrared Spectroscopy (NIR) used to determine the concentration of substances in solid and liquid samples, which an optical condenser with fixed lenses was developed in order to increase the Signal-Noise relation, also LEDs were used as a light source and InGaAsSb sensors, to detect diffusedly reflected light. It mentions that the instrument described in the article was tested in samples of milk and water mixtures, achieving an efficiency of approximately $99 \%$. In [7] they use a similar method and mention hat spectroscopy is an alternative solution to detect the addition of water in milk, the method proposed is based on diffuse reflectance, the electronic device created has a reflectance of $88 \%$, using infrared LEDs and a photodiode In-Ga-As-Sb.

In [8] they mention that milk analysis is an important role in determining its quality; therefore, in the article they describe the $\mathrm{pH}$ behavior of milk, in addition to its density. The impact of the addition of water on the milk produces a change in the $\mathrm{pH}$ electrode potential by approximately $1 \mathrm{mV}$ and the density varies by $0.004 \mathrm{~kg} / \mathrm{m} 3$.

In the research [9] a system was designed to measure milk adulteration, the system shows an accuracy of approximately $95 \%$ with a deviation of $1 \%$; The system was tested to measure the percentage of fats and water; another addition to the system is the heater interface, which it seeks to perform tests for the ultrasonic sensor by transporting the cold samples to environmental temperature, to obtain greater precision.

In [10] there is a need for new and more robust techniques for the detection of adulterated milk; therefore, they propose a prototype of a digital, microcontrolled and portable photometer, which uses three infrared LEDs, with this equipment, they measure the transmittance of infrared radiation in milk samples, which evaluate whether it has water or not. The team demonstrated an average absolute error less than $1 \%$.

In [11] they mention that the electrical conductivity of milk is frequently used for diagnostic tests of milk quality, in addition the electrical conductivity of milk can be an indicator of mastitis, which is a disease of cattle, and as a method to 
measure the amount of water, present in milk. Therefore, its objective is to design a system that allows to detect the percentage of water in milk and subsequently predict the presence of mastitis.

In [12], a non-contact method for the detection of milk adulteration is proposed, considering approximately 500 samples of milk in three categories, cow's milk, buffalo milk, skim milk; the parameter involved in the detection was the refractive index, which changes as the amount of water present in the milk changes. According to the results shown, the accuracy was approximately $95 \%$.

Section II presents the methodology that has been followed for the research work. Section III contains the preliminary results obtained. In Section IV are the discussions and finally in the section, the conclusions of the research is presented.

\section{Methodology}

For the present work, the methodology to follow is schematized in the block diagram shown in Fig. 1.

\section{A. Acquisition of Images}

For the image acquisition stage, it makes mixtures of fresh milk and water according to Table I, mixtures are made in a $100 \mathrm{ml}$ beaker, which is placed on a dark surface, pointed to the liquid with a laser and a photo is captured, it should be taken into account that the photos are taken at an approximate distance of $7 \mathrm{~cm}$ from the glass; in total, 675 images were acquired for each mixture of milk and water.

From Table I, it obtains 11 classes for the processing and classification stage which are named as: M100W00, M90W10, M80W20, M70W30, M60W40, M50W50, M40W60, M30W70, M20W80, M10W90 and M00W100, these names correspond to the labels that will be placed on each image.

\section{B. Image Processing}

For this stage the Thresholder color tool was used as the reader can see in Fig. 2, in which it selected four areas of interest, in which variations of the color are presented, due to the interaction of the laser with the mixture, which produces a superficial diffuse reflection, producing in the photographs zones differentiated by color; a mask is created for each zone and the code generated by the application is imported.

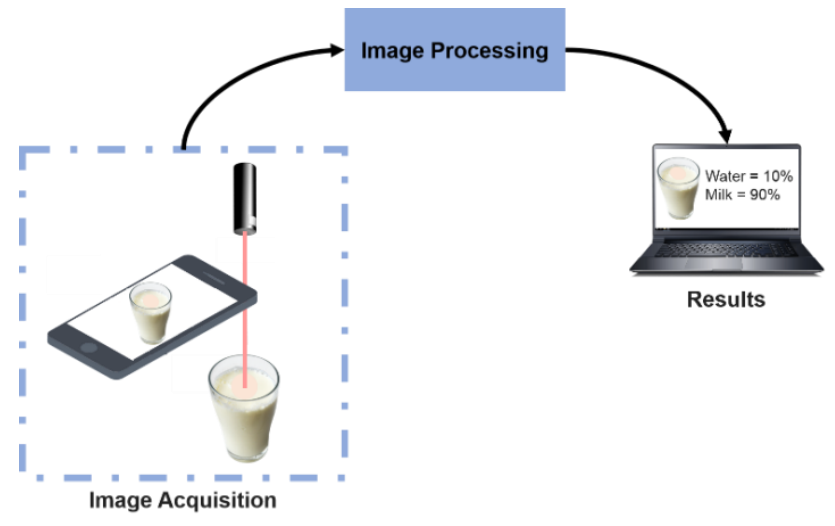

Fig. 1. Work Scheme.
TABLE. I. CHART OF MILK AND WATER PROPORTIONS

\begin{tabular}{|l|l|l|l|l|}
\hline Milk (ml) & Water $(\mathbf{m l})$ & & Milk $(\mathbf{m l})$ & Water $(\mathbf{m l})$ \\
\hline 100 & 0 & 40 & 60 \\
\hline 90 & 10 & 30 & 70 \\
\hline 80 & 20 & 20 & 80 \\
\hline 70 & 30 & 10 & 90 \\
\hline 60 & 40 & 0 & 100 \\
\hline 50 & 50 & & \\
\hline
\end{tabular}

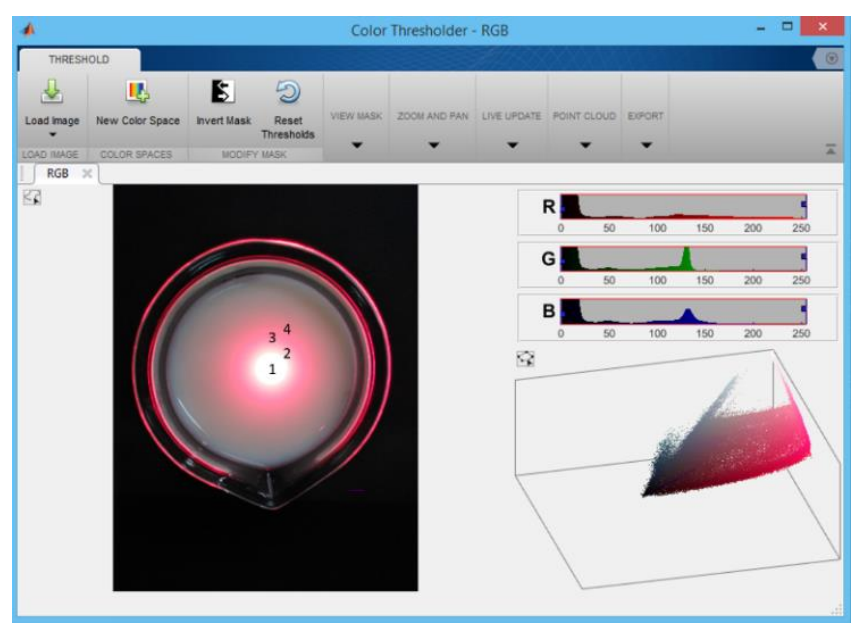

Fig. 2. Thresholder Color Tool.

In order to perform the same procedure on all images, an algorithm was created, of which it can see its general scheme in Fig. 3.

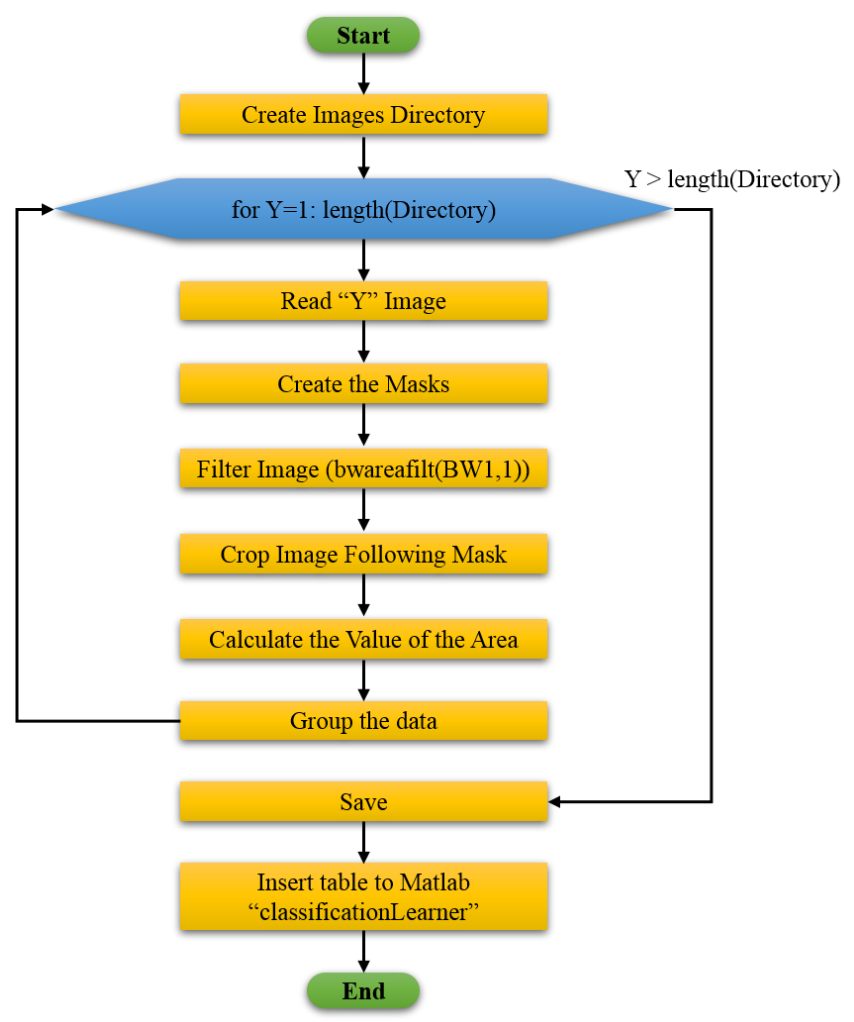

Fig. 3. Scheme of Data Processing. 
Fig. 3 shows the procedure to create masks, filter and crop the image, for the four zones mentioned above, of which the corresponding area is calculated, which will be a characteristic for the classification stage with the Classification Learner application.

\section{RESULTS}

In Fig. 4(a), it can see an original image which is the milk concentration equivalent to $30 \%$ that is $30 \mathrm{ml}$ of milk and $70 \mathrm{ml}$ of water, in Fig. 4(b), 4(c), 4(d) and 4(e) are cropped images corresponding to the four characteristics previously missioned.
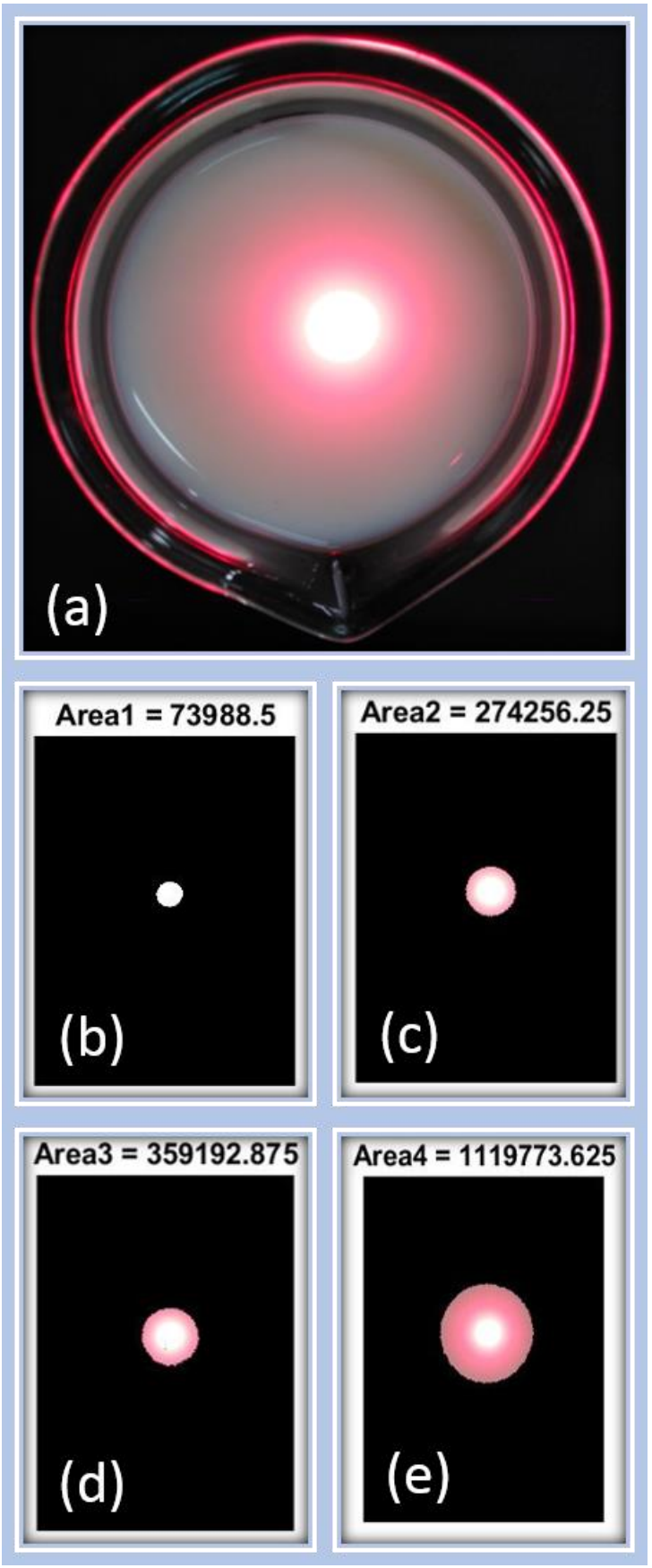

Fig. 4. (a) Photograph of Adulterated Milk; (b) Cropping of the Central Zone; (c) Cropping the Second Zone (d) Cropping the Third Zone

(e) Cropping the Fourth Zone.

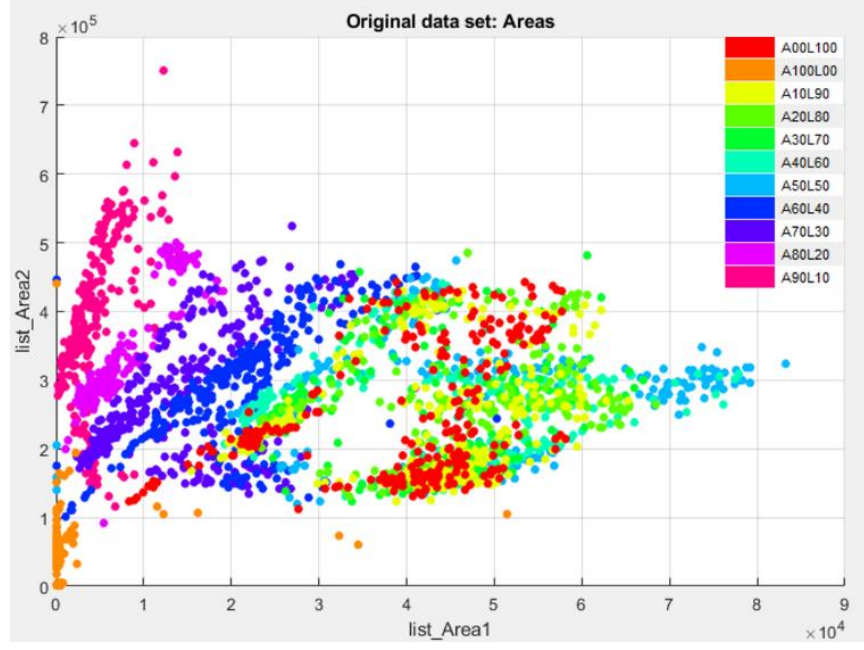

Fig. 5. Graphing the Data in the Classification Learner Application.

\begin{tabular}{|c|c|c|c|c|c|c|c|c|c|c|c|}
\hline $\mathrm{A} 00 \mathrm{~L} 100$ & 628 & & 10 & 8 & 8 & 10 & 5 & 5 & & & 1 \\
\hline A100L00 & 2 & 666 & 1 & & & & 1 & 3 & 2 & & \\
\hline A10L90 & 12 & & 632 & 9 & 9 & 11 & 1 & 1 & & & \\
\hline A20L80 & 8 & & 12 & 637 & 3 & 9 & 4 & 2 & & & \\
\hline A30L70 & 7 & & 17 & 9 & 620 & 13 & 7 & 2 & & & \\
\hline A40L60 & 2 & & 8 & 7 & 10 & 640 & 3 & 5 & & & \\
\hline A50L50 & 3 & & 2 & 3 & 4 & 9 & 647 & 5 & 2 & & \\
\hline$A 60\llcorner 40$ & 2 & & & 1 & 1 & 5 & 7 & 647 & 9 & & 3 \\
\hline A70L30 & 1 & 1 & & & & & & 33 & 691 & 19 & \\
\hline A80L20 & & & & 1 & & & & & 3 & 601 & \\
\hline A90L10 & & & & & & & & & & & 675 \\
\hline
\end{tabular}

Fig. 6. Confusion Matrix Generated by the Classification Learner Application.

All the file of characteristics because of the algorithm described in the previous section, are introduced in a Matlab tool called Classification Learner, which generates a graph of the data as it shows in Fig. 5. The fine k-nearest neighbors (KNN) algorithm was chosen to classify the data.

Using the aforementioned algorithm, the application generates an approximate success rate of $95.4 \%$, also generate a confusion matrix as the reader can see in Fig. 6, which the performance of the chosen algorithm is evaluated.

\section{DISCUSSION AND EVALUATION}

For the detection of adulterated milk with water, there are many jobs as in [5] where an efficiency of $95 \%$ was obtained through the study of the density of the sample; as well as in [6], where a $99 \%$ success rate was obtained using sensors and infrared light; in [9] they show an accuracy of approximately 
$95 \%$ with a deviation of $1 \%$; there are research as in [2] that by carrying out a study of the electrical impedance they obtain an efficiency of $94.9 \%$ applying the KNN algorithm; in [12] a distinction is made between milk of three categories, cow's milk, buffalo milk, skim milk, obtaining an accuracy of approximately 95\%; finally in [13] they mention that they have obtained an efficiency percentage of $83 \%$ using neural networks; in our case, it obtains similar results by applying the KNN method, image processing and Classification Learner application, which it obtains efficiency in the classification of $95.4 \%$.

It is important to mention that no papers related to the study of milk adulteration were found, through image analysis, references obtained and compared in this section, are papers that at least are related to the algorithm applied for the classification stage.

\section{CONCLUSIONS}

Image treatment is widely applied nowadays in different industries, in this document it demonstrates that it can apply image treatment to detect the amount of water presented in a $100 \mathrm{ml}$ sample of milk, only by pointing to the sample with a laser and taking a photography, it is shown that the procedure used has a very good efficiency, equivalent to other methods of detecting milk adulteration.

Future work, the main action is in improving the efficiency and the proposed method, studying whether the laser power influences the calculations performed as well as the classification; the main difficulty of this paper is that it cannot detect adulterated milk with substances other than water, for this reason the possibility of conducting studies that identify compounds other than water using a method equal or similar to that proposed in this work remains open for future works.

\section{REFERENCES}

[1] S. Das, M. Sivaramakrishna, B. Goswami, and K. Biswas, "Study of Electrical Equivalent Model of The PMMA Coated Probe Dipped In Milk And Milk Adulterated With Tap Water," Int. Conf. Ind. Electron. Control Robot., pp. 53-58, 2010.
[2] G. Durante, W. Becari, F. A. S. Lima, and H. E. M. Peres, "Electrical Impedance Sensor for Real-Time Detection of Bovine Milk Adulteration,” IEEE Sens. J., vol. 16, no. 4, pp. 861-865, 2016.

[3] T. K. Mondal and S. Das, "Discrimination of various Milk adulterated sample using Linear Discrimination Analysis(LDA)," 11th IEEE India Conf. Emerg. Trends Innov. Technol. INDICON 2014, 2014.

[4] M. Sude and K. Ghodinde, "Electrical impedance Sensor for Real-Time Detection of Urea and Starch in Milk," 2019 3rd Int. Conf. Trends Electron. Informatics, no. Icoei, pp. 431-434, 2019.

[5] P. S. Venkateswaran, A. Sharma, S. Dubey, A. Agarwal, and S. Goel, "Rapid and Automated Measurement of Milk Adulteration Using a 3D Printed Optofluidic Microviscometer (OMV)," IEEE Sens. J., vol. 16, no. 9, pp. 3000-3007, 2016.

[6] L. De Souza Ribeiro, F. A. Gentilin, J. A. De França, A. L. De Souza Madureira Felício, and M. B. M. De França, "Development of a hardware platform for detection of milk adulteration based on nearinfrared diffuse reflection," IEEE Trans. Instrum. Meas., vol. 65, no. 7, pp. 1698-1706, 2016.

[7] L. Da Silva Dias, J. C. Da Silva, A. L. De Souza Maudeira Felicio, and J. A. De Franca, "A NIR Photometer Prototype with Integrating Sphere for the Detection of Added Water in Raw Milk," IEEE Trans. Instrum. Meas., vol. 67, no. 12, pp. 2812-2819, 2018.

[8] R. B. Somasagar and A. Kuasagur, "Potentiometrie and $\mathrm{pH}$ based electronic method for dilution detection in milk," 2017 Int. Conf. Intell. Comput. Instrum. Control Technol. ICICICT 2017, vol. 2018-Janua, pp. 1762-1765, 2018.

[9] A. Dave, D. Banwari, S. Mansinghani, S. Srivastava, and S. Sadistap, "Ultrasonic sensing system for detecting water adulteration in milk," IEEE Reg. 10 Annu. Int. Conf. Proceedings/TENCON, pp. 2228-2231, 2016.

[10] M. Moreira et al., "A Low-Cost NIR Digital Photometer Based on InGaAs Sensors for the Detection of Milk Adulterations with Water," IEEE Sens. J., vol. 16, no. 10, pp. 3653-3663, 2016.

[11] L. M., Alonso V., and A. M. M., "Design and construction of a system for measuring the concentration of water in milk," Proc. - Electron. Robot. Automot. Mech. Conf. CERMA 2006, vol. 2, pp. 47-51, 2006.

[12] A. Dave, D. Banwari, S. Srivastava, and S. Sadistap, "Optical Sensing System for Detecting Water Adulteration in Milk," IEEE Reg. 10 Annu. Int. Conf. Proceedings/TENCON, pp. 2228-2231, 2016.

[13] M. A. Putra, M. Rivai, and A. Arifin, "Milk Assessment using Potentiometric and Gas Sensors in Conjunction with Neural Network," Proceeding - 2018 Int. Semin. Intell. Technol. Its Appl. ISITIA 2018, pp. 409-412, 2018. 